\title{
Editorial
}

\section{Molecular Pathways in Normal Aging and Neurodegeneration: Mechanisms and Therapeutics}

Dedicated in honor of Dr. Arturo D. Melo S., who inspired the building of neuroscience research in the Republic of Panama

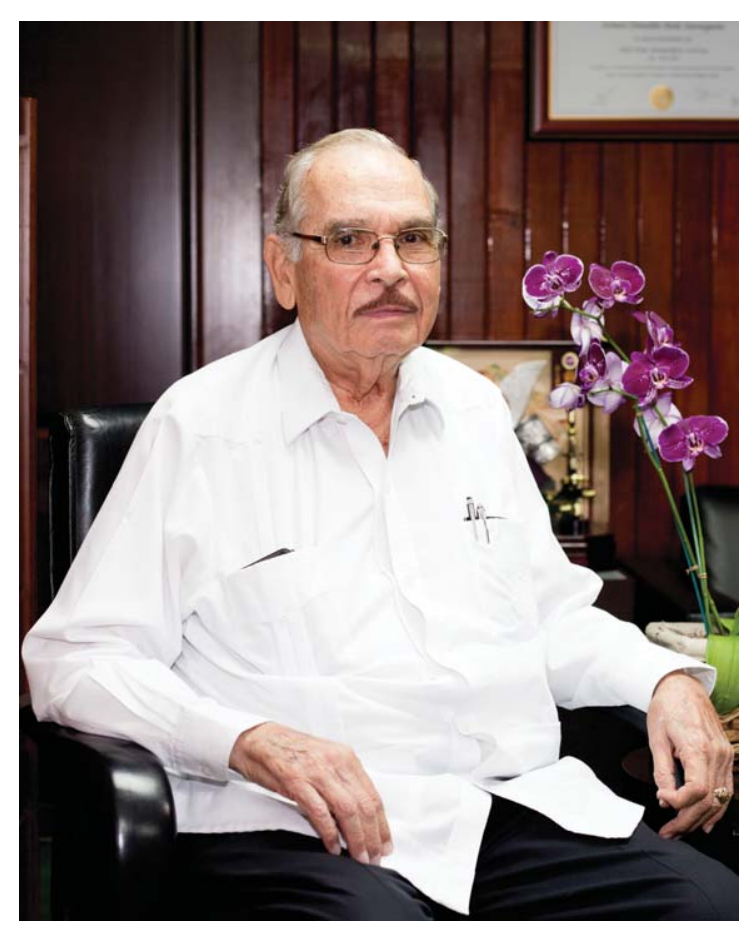

The brain is the most complex organ of the body whose structure and function in normal aging and in age-related brain disorders is little understood. The adult healthy brain has 100 billion neurons, and each neuron has a long axon with branching extensions. The neurons communicate through extensions via synaptic junctions. Interestingly, the adult brain contains about 100 trillion synapses. Synaptic plasticity is very crucial for healthy brain aging and any malfunction in synaptic biology leads to age-related neurodegenerative disorders. However, the biology of neurodegenerative disorders is challenged with complex etiology and pathology. There are no specific biomarkers and the biochemical targets for drug discovery for neurodegeneration are controversial. For example, accumulation of the proteins/peptides such as amyloid- $\beta$, tau, synuclein, prion, and others in different degenerative diseases is still not equivalent to disease. Furthermore, degenerating brains show oxidative stress, inflammation, DNA damage, extensive shrinkage from cell loss, and accumulation of debris from dead or dying neurons. It is very interesting to mention that changes associated with Alzheimer's disease may begin 20 or even more years before symptoms appear and the same is true in other neurodegenerative conditions. Resolving this issue by early diagnosis of the disease through research on biomarkers/imaging is a hot topic. The complex issue is, as neuronal damage increases, the brain cannot compensate for the changes due to biochemical repair failure. The resulting failure leads to increasing cognitive decline including symptoms such as memory loss or confusion. Finally, basic bodily functions such as swallowing are impaired.

The number of Alzheimer's or other dementia pathologies continues to increase as the population ages. It is estimated that the affected world population is currently 48 million and it is predicted to almost double by 2050 . In 2010, there were 454,000 new cases of Alzheimer's dementia, and it is predicted that by 2030 , that number will grow to 615,000 (a $35 \%$ increase from 2010). By 2050, the number of 
people in the US aged 65 and older with Alzheimer's dementia may triple, increasing from 5.3 million to 13.8 million. This situation is alarming as it is going to affect health care systems, bringing about a social and economic impact on both developing and developed countries.

It is time to make discoveries on the complex etiology, find novel biomarkers, and reveal new biochemical pathways for neuronal cell death and new drug targets. In this supplementary issue of the Journal of Alzheimer's Disease, we focus on Molecular Pathways in Normal Aging and Neurodegeneration: Mechanisms and Therapeutics, inviting articles from leading neuroscientists representing the scope of the latest advances in the field. We covered topics such as 1) resveratrol and curcumin combination effect against aluminum chloride-induced neuroinflammation; 2) phytochemical ginkgolide B attenuates $A \beta_{1-42}$ induced oxidative damage; 3 ) the denervation of neuromuscular junctions and axonal degeneration in transgenic mice expressing mutant (P301S) tau protein; 4) novel curcumin derivatives for the prevention of the inflammatory process in Alzheimer's disease; 5) post-translational oxidative modifications of mitochondrial complex I (NADH: ubiquinone oxidoreductase); 6) cognitive deficits after cerebral ischemia and underlying dysfunctional plasticity; 7) debate on DNA damage response in neurons; 8) new evidence on chromatin-bound oxidized $\alpha$-synuclein causes strand breaks in neuronal genomes; 9) a debate on Chinese medicine: a hope for neurodegenerative diseases; 10) neuroinflammation and hippocampal neurogenesis; 11) human immunodeficiency virus type 1-associated common neurological complications; 12) ApoE and associated gene polymorphism as a potent biomarker identifying the susceptible individuals for Alzheimer's disease; 13) fenugreek seed powder attenuated aluminum chloride induced tau pathology; and 14) resveratrol intervenes cholesterol and isoprenoids mediated amyloidogenic processing of $\mathrm{A} \beta \mathrm{PP}$ in familial Alzheimer's disease. In overview, you can recognize that this supplementary issue contains novel pathways, debates on new mechanisms, novel molecular targets, novel neuroprotective molecules, DNA damage, neuroimmunology and neurogenesis, epigenetic links, and axonal degeneration.

We dedicate this issue to Dr. Arturo D. Melo S, a business man with great scientific spirit in aging research. His philanthropic donation starting back in
2011 inspired us to initiate neuroscience research in the Republic of Panama and helped to build an International Neuroscience Centre in collaboration with different world leading centers. Dr. Arturo Melo has great interest in unraveling new discoveries in the aging process and age-related diseases and he has encouraged young minds to pursue molecular aspects of brain research. Dr. Arturo Melo is a man of great inspiration and was honored for his philanthropic activity by Nobel Laureate Dr. Robert Huber in the Republic of Panama in 2016.

We hope this supplementary issue will be an inspiring guide for young scientists who would like to enter into the neuroscience field and also be a valuable contribution to scientists working in the hot spots of neurodegenerative pathways and drug discovery. We thank all the experts for their enthusiastic contributions and thank particularly Beth Kumar, Managing Editor, Journal of Alzheimer's Disease for her support. We also profoundly thank the reviewers for their timely support.

\section{GUEST EDITORIAL TEAM:}

Prof. K.S. Jagannatha Rao, Director, Centro de Neurociencias, Instituto de Investigaciones Científicas y Servicios de Alta Tecnología, City of Knowledge, Republic of Panama.

Prof. George Perry, Dean and Professor of Biology, College of Sciences, University of Texas at San Antonio, San Antonio, TX, USA.

Prof. Y.Z. Zhu, Chair Professor, Director, School of Pharmacy, Macau University of Science and Technology, Macau, China.

Prof. Muralidhar L. Hegde, Associate Professor, Department of Radiation Oncology, Institute for Academic Medicine, Associate Member, Houston Methodist Neurological Institute, Associate Professor of Neurology, Weill Medical College of Cornell University, New York, Houston Methodist Hospital, Houston, TX, USA.

Prof. Thomas von Zglinicki, Scientific Director, Newcastle University Institute for Ageing, Newcastle University, Newcastle upon Tyne, UK.

Prof. Miguel A. Perez-Pinzon, Professor of Neurology \& Neuroscience, Vice-Chair for Basic Science (Neurology), Director, Cerebral Vascular Disease Research Laboratories, Dept. of Neurology, University of Miami Miller School of Medicine, Miami, FL, USA. 